\title{
Variação morfométrica em Bothropoides jararaca (Serpentes, Viperidae) no Rio Grande do Sul
}

\author{
Nathalia Rocha Matias ${ }^{1,2}$, Maria Lúcia Machado Alves ${ }^{1}$, Moema Leitão de Araujo ${ }^{1} \&$ Diego Marques \\ Henriques Jung ${ }^{2}$
}

1. Núcleo Regional de Ofiologia de Porto Alegre (NOPA), Museu de Ciências Naturais, Fundação Zoobotânica do Rio Grande do Sul. Rua Dr. Salvador França, 1427, bairro Jardim Botânico,
90690-000, Porto Alegre, RS, Brasil. (nathimatias@yahoo.com.br)
2. Universidade Luterana do Brasil. Av. Farroupilha, 8001, bairro São José, 92425-900, Canoas, RS, Brasil.

\begin{abstract}
Morphometric variation in Bothropoides jararaca (Snakes, Viperidae) in Rio Grande do Sul. Although male and female snakes are born with the same size, growth rates and age of sexual maturation may be different, thus determining sexual dimorphism in the later stages of life. We evaluated the incidence of sexual and ontogenetic morphometric variation in Bothropoides jararaca (Wied, 1824), exploring relationships involving body size and sexual maturity through 14 morphometric variables. We analyzed 142 specimens from the state of Rio Grande do Sul, southern Brazil. Morphometric data - head length, snout-vent length, tail length, total length; head width, ocular width, nasal-loreal width, tail width; nasal-ocular distance, ocular-loreal distance, loreal-nasal distance, ventral-sinfisal distance and rostral-labial distance - and length/ diameter of ovarian follicles, were taken in millimeters by simple rule and analogical caliper, precision $0.05 \mathrm{~mm}$. Sex determination was performed by inspection of the gonads. Age was associated to sexual maturity. For the statistical analysis the analysis of variance (ANOVA) with Tukey's post hoc test was used with, linear regression and canonical discriminant analysis (CDA). Most measures indicated sexual dimorphism (ANOVA, $P<0.05$ ) only in adults (Tukey, $P<0.05$ ). Regression analysis showed that snout-vent length explains the behavior of other variables $(P<$ 0.001 ) and in all measures, females grow more than males. The ADC has been successful in separating the genders and age groups, with biological meaning, whereas $79.2 \%$ of cases were correctly classified.
\end{abstract}

KEYWORDS. Ontogeny, morphology, dimorphism.

RESUMO. Apesar de machos e fêmeas de serpentes nascerem com o mesmo tamanho, as taxas de crescimento e a idade da maturação sexual podem ser diferentes, determinando dimorfismo sexual em estágios posteriores da vida. Avaliamos a ocorrência de variação morfométrica sexual e ontogenética em Bothropoides jararaca (Wied, 1824), explorando as relações entre tamanho corporal e amadurecimento sexual através de 14 variáveis morfométricas. Foram analisados 142 espécimes provenientes do estado do Rio Grande do Sul, sul do Brasil. Os dados morfométricos - comprimento da cabeça, rostro-cloacal, da cauda, comprimento total; largura da cabeça, ocular, nasal, loreal, da cauda; distância ocular-nasal, ocular-loreal, loreal-nasal, ventral-sinfisal e rostral-labial - e comprimento/diâmetro dos folículos ovarianos, foram tomados em milímetros, através de régua simples e paquímetro analógico de precisão $0,05 \mathrm{~mm}$. A determinação sexual foi realizada por inspeção das gônadas. A classificação etária foi associada à maturidade sexual. Para as análises estatísticas foram utilizadas análises de variância (ANOVA) com teste de Tukey post hoc, regressão linear e análise discriminante canônica (ADC). A maioria das medidas indicou dimorfismo sexual $($ ANOVA, $P<0,05)$ apenas em adultos (Tukey, $P<0,05$ ). As análises de regressão mostram que o comprimento rostro-cloacal explica o comportamento das demais variáveis $(P<0,001)$ e que em todas as medidas as fêmeas crescem mais que os machos. A ADC foi exitosa em separar as classes sexuais e etárias, apresentando significado biológico, considerando $79,2 \%$ dos casos como corretamente classificados.

PALAVRAS-CHAVE. Ontogenia, morfometria, dimorfismo.

No Brasil, Viperidae está representada por 28 espécies, onze atualmente alocadas em Bothropoides (SBH, 2010). Este gênero foi recentemente estabelecido por FENWICK et al. (2009), em estudo baseado em caracteres morfológicos e moleculares que propõem um novo arranjo taxonômico de Bothrops, Bothriopsis e Bothrocophias. Bothropoides jararaca (Wied, 1824) apresenta hábito terrestre e semiarborícola, com atividade basicamente noturna. Sua dieta apresenta variação ontogenética, já que indivíduos jovens alimentam-se de quilópodes, lagartixas, filhotes de roedores e principalmente anuros, enquanto os adultos geralmente predam roedores. Bothropoides jararaca é vivípara e gera de 5 a 16 filhotes (SAZIMA, 1992; HarTMAnN et al., 2003). Segundo Lema (1994), no Rio Grande do Sul, é muito frequente nas poucas regiões florestadas do norte, atingindo a Encosta Inferior do Nordeste até Porto Alegre.

O dimorfismo sexual em serpentes pode ser detectado em relação às dimensões e proporções corporais, ao número ou formas das escamas e posição e/ou tamanho de órgãos ou glândulas (SHINE, 1993; Kissner et al., 1998; Keogh \& Walach, 1999).
Diferenças no tamanho corporal entre machos e fêmeas foram registradas para várias espécies de serpentes brasileiras (SBH, 2007). Embora machos e fêmeas nasçam com o mesmo tamanho, podem apresentar taxas de crescimento e a idade da maturação sexual diferentes, demonstrando dimorfismo sexual em estágios posteriores da vida (SHINe, 1990). Estudos sobre dimorfismo sexual realizados com indivíduos de Colubridae e Dipsadidae revelaram maior comprimento rostro-cloacal (CRC) em fêmeas (FITcH, 1981; SHINE, 1994; JORDÃo \& BiZERRA, 1996) e maior comprimento de cauda (CC) em machos (Klauber, 1943; Clark, 1966; KinG, 1989; Santos JR \& Ribeiro, 2005;). Geralmente as fêmeas apresentam maior comprimento total (CT) que os machos, o que pode estar relacionado com seu sucesso reprodutivo (Semlitsch \& GibBons, 1982; Shine, 1994; Zug, 2001). Em Bothrops moojeni Hoge, 1966 e Rhinocerophis alternata (Duméril, Bibron \& Duméril, 1854), os dados biométricos demonstraram maior tamanho cefálico nas fêmeas, o que pode estar relacionado ao maior tamanho das suas glândulas de peçonha. (FARIA \& Brites, 2003; Mesquita \& Brites, 2003). 
Diferenças no tamanho da cauda também são comuns, indicando que os machos portam caudas mais longas para acomodar o hemipênis e os músculos retratores (KLAUBER, 1943; ClARK, 1966; KING, 1989).

JANEIRO-CINQUINI et al. (1990) e JANEIRO-CINQUINI (2004) verificaram correlação positiva entre o tamanho da ninhada e o comprimento rostro-cloacal (CRC) da fêmea de Bothropoides jararaca. SolóRzAnO \& CERDAs $(1987,1989)$ encontraram a mesma correlação positiva em Caudisona durissa (Linnaeus, 1758) e Bothrops asper (Graman, 1883) da Costa Rica. No estudo anatômico de Gomes \& Puorto (1993) foram observadas diferenças na folidose de $B$. jararaca: a região correspondente aos ovários conteve maior número de escamas ventrais quando comparada à correspondente aos testículos, assim como as escamas subcaudais, que foram mais numerosas em machos.

$\mathrm{O}$ estudo da biologia reprodutiva de Botropoides jararaca em cativeiro demonstrou que no primeiro ano de vida as fêmeas crescem mais rapidamente que os machos (Alves et al., 2000). Entretanto, dos 12 aos 18 meses houve inversão desta relação e machos apresentaram maior comprimento que as fêmeas. Somente entre os 18 e 42 meses de vida as fêmeas voltaram a apresentar maiores CRCs.

O dimorfismo sexual em Bothropoides jararaca foi demonstrado por JANEIRO-CINQUINI et al. (1992), que registraram cauda com maiores dimensões nos machos e maior comprimento total nas fêmeas. Entretanto, neste estudo não foram consideradas as possíveis influências da distribuição geográfica e classes etárias na ocorrência do dimorfismo morfométrico, recomendadas por StAMPS (1993). MARTINS et al. (2001), em estudo envolvendo vinte espécies de Bothrops, constataram a influência do uso do substrato no tamanho da cauda e forma do corpo dessas serpentes, demonstrando a importância dos agentes seletivos nas diferenças morfométricas interespecíficas.

O objetivo deste trabalho é verificar a ocorrência de variação morfométrica sexual e ontogenética em Bothropoides jararaca utilizando 14 variáveis morfométricas e avaliar se existe dimorfismo sexual para estas variáveis em todas as classes etárias.

\section{MATERIAL E MÉTODOS}

A amostra estudada compreende 142 exemplares (71 fêmeas e 71 machos) provenientes de 42 municípios do estado do Rio Grande do Sul e, eventualmente, espécimes provenientes de resgates de fauna de barragens implementadas em quatro municípios do estado de Santa Catarina. Estes municípios integram sete regiões fitoecológicas: Área de formações pioneiras, Área de tensão ecológica, Floresta ombrófila densa, Floresta ombrófila mista, Floresta estacional decidual, Floresta estacional semidecidual e Savana. Todos exemplares estão depositados na Coleção de
Répteis do Museu de Ciências Naturais (MCN), Fundação Zoobotânica do Rio Grande do Sul, Porto Alegre, RS.

Para evitar a influência do cativeiro, foram analisados os espécimes mortos logo após a captura ou que viveram, no máximo, um mês em cativeiro. A determinação sexual foi realizada por inspeção das gônadas, através de incisão ventral com bisturi, sendo considerados maduros os machos de CRC igual ou maior que o menor macho portador de canais deferentes enovelados e opacos (SHINe, 1977a), e as fêmeas de comprimento rostro-cloacal (CRC) igual ou maior que a menor fêmea portadora de folículos em vitelogênese secundária $(>10 \mathrm{~mm})$, ou com embriões (SHINE, 1977b). A classificação etária foi associada à maturidade sexual e ao tamanho (comprimento rostro-cloacal, CRC). Foram considerados adultos os indivíduos reprodutivamente maduros, jovens aqueles com CRC menor que o apresentado pelo menor indivíduo maduro e filhotes indivíduos com CRC até 400 e $405 \mathrm{~mm}$ para machos e fêmeas, respectivamente. Para filhotes estes CRCs foram estabelecidos para não perder modificações morfométricas importantes no desenvolvimento dos animais, já que os indivíduos apresentam grande diferença de tamanho desde quando nascem até atingirem a maturidade.

Os dados morfométricos - comprimento rostro-cloacal (CRC), comprimento da cauda (CC), comprimento total (CT), largura da cauda (LC) (Fig. 1); largura da cabeça (LCA), largura ocular (LO), largura nasal (LN), largura loreal (LL) (Fig. 2); distância ocularnasal (DON), distância ocular-loreal (DOL), distância loreal-nasal (DLN) (Fig. 3); comprimento da cabeça (CA), distância rostral-labial (DRL) (Fig. 4); distância ventral-sinfisal (DVS) (Fig. 5) - e comprimento/diâmetro dos folículos ovarianos foram aferidos em milímetros, através de régua simples e paquímetro analógico de precisão $0,05 \mathrm{~mm}$. As análises estatísticas foram conduzidas através de métodos que permitem explorar a variação entre os grupos (classes etárias e sexuais).

Para comparação das variâncias das variáveis morfométricas entre as classes etárias e sexuais utilizaram-se análises de variância (ANOVA) com teste de Tukey post hoc. Com intuito de investigar a influência geral do tamanho (CRC) nas demais variáveis utilizou-se análises de regressão linear, testando-se o

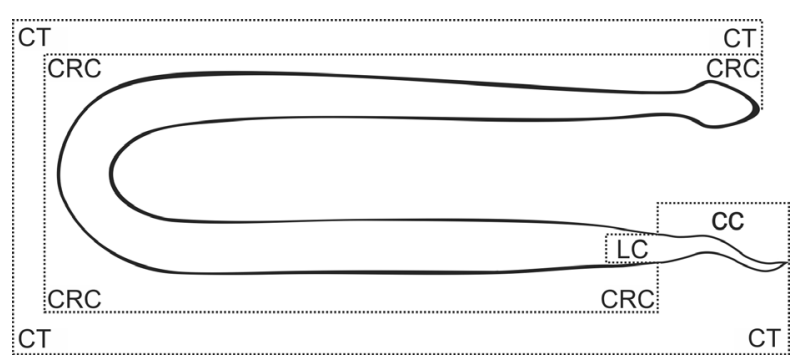

Fig. 1. Variáveis morfométricas em Bothropoides jararaca (Wied, 1824) (LC, largura da cauda; CC, comprimento da cauda; CRC, comprimento rostro-cloacal; CT, comprimento total). 


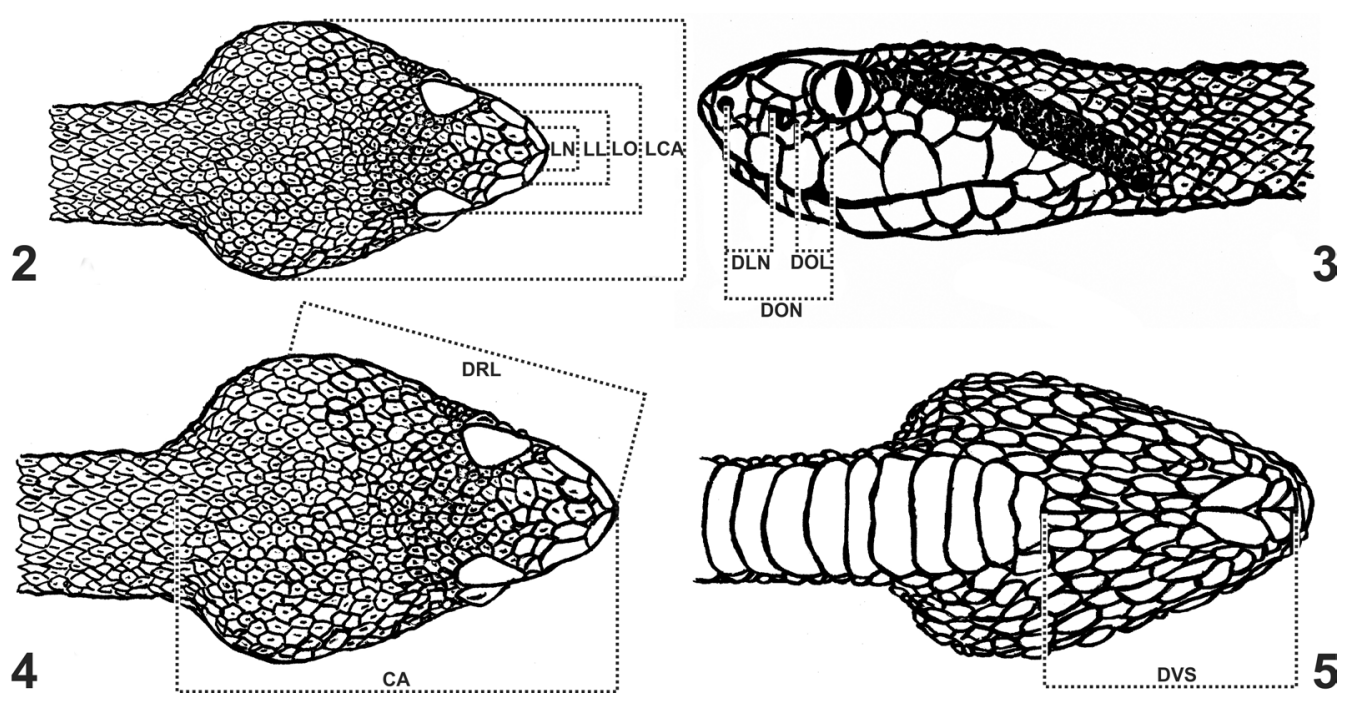

Figs. 2-5. Variáveis morfométricas em Bothropoides jararaca (Wied, 1824): 2, 4, cabeça, vista dorsal (LN, largura nasal; LL, largura loreal; LO, largura locular; LCA, largura da cabeça; DRL, distância rostral labial; CA, comprimento da cabeça); 3, cabeça, vista lateral (DLN, distância loreal nasal; DOL, distância ocular loreal; DON, distância ocular nasal); 5, cabeça, vista ventral (DVS, distância ventral sinfisal).

comportamento das variáveis para machos e fêmeas separadamente. Para as técnicas univariadas as probabilidades foram consideradas significativas para valores de $P$ menores que 0,05 . A técnica multivariada de análise discriminante canônica (ADC) foi realizada para testar a efetividade da classificação da amostra nas classes etárias e sexuais, através das variáveis morfométricas, assim como para identificar as variáveis mais eficientes na discriminação dos grupos.

Material examinado. BRASIL, Rio Grande do Sul: Anta Gorda, $2 \widehat{\jmath}$ (MCN 11170, 11219); Barracão, 2 \% (MCN 9210, 13607); Caxias do Sul, 4 đ (MCN 1074, 1075, 1076, 1078); Candelária, o (MCN 7366); (Botucaraí), o (MCN 13988); Canela, $\widehat{o}$ (MCN 2764), 오 (MCN 13420); Cazuza Ferreira, ô (MCN 5524); Dois Irmãos, $2 \widehat{\jmath}$ (MCN 7583, 11189); Estância Velha, đ̂ (MCN 9020); Fagundes Varela, ô (MCN 10061); Garibaldi, 29 के (MCN 9289, 9490, 9491); Gramado, 2ㅇ, 30े (MCN 6828, 8430, 8445, 8974, 10641); Igrejinha, 3 ㅇ (MCN 9425, 11389, 11392); Iraí $q$ (MCN 5881); Ivoti, 2 ( $\mathrm{MCN} 7705,8160)$; Machadinho, 3ㅇ, 2 đ (MCN 13360, 13406, 13408, 14039, 14084); Maquiné, $3 \hat{\circ}$ (MCN 8811, 12108, 12112); Maximiliano de Almeida, $2 \widehat{\jmath}$ (MCN 13361, 13407); Nova Petrópolis, 13우 $10{ }^{\Uparrow}$ (MCN 9442, 9576, 9611, 9637, 9638, 9800, 9817, 10008, 10009, 10010, 10011, 10313, 10319, 10351, 10586, 10591, 11092, $11369,11451,11796,14358,14753,14758$ ); Novo Hamburgo, o (MCN 4309); Osório, ㅇ (MCN 10590); Paim Filho, o (MCN 6167), 우 (MCN 6299); Pinhal da Serra, ô (MCN 16140); Pinhal Grande, 우 (MCN 13153); Porto Alegre, 4ㅇ, $3{ }^{\lambda}$ (MCN 1810, 3506, 3650, 7433, 10000, 13449, 14972); Putinga, 우 (MCN 9349), ô (MCN 9350); Rolante, ô (MCN 8655); Salvador do Sul, 3今, o (MCN 10318, 11681, 12124, 12914); Santo Antônio da Patrulha, 39 (MCN 10700, 13158, 15036); São Francisco de Paula, 4ㅇ, $2 \widehat{\jmath}$ (MCN 4297, 5454, 7419, 7850, 9974, 10001); São José do Sul, 3 đ (MCN 14160, 14658, 15741); São Leopoldo, $2 \widehat{O}$ (MCN 2101, 8512); Sapiranga, o (MCN 10035); Taquara, ô (MCN 9159); Tenente Portela, $\hat{o}$ (MCN 6612), 오 (MCN 7134); Terra de Areia $2 \hat{\jmath}$ (MCN 25, 10999); Torres $5 \hat{0}$, 우 (MCN 9118, 9119, 9120, 9140, 9802, 9881); Tucunduva, 2 으 (MCN 14317, 15367); Tuparendi, 2o, ô (MCN 10290, 12001, 12136); Veranópolis, ô (MCN 10812), + (MCN 14891); Viamão, 3 \%, (MCN 8274, 7982, 8391, 9985); Victor Graeff, ㅇ (MCN 9774), o (MCN 9775). Santa Catarina: $q$ (MCN 15766), o (MCN 16285); Anita Garibaldi, 5우, 3 ` (MCN 15632, 15633, 15634, 15635, 15653, 15655, 15767, 16364); Campo Belo do Sul, ㅇ (MCN 15768); Campos

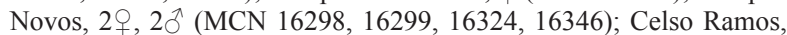
3 ㅇ, $2 \overbrace{}^{\lambda}(\mathrm{MCN} 15388,16326,16327,16361)$.

\section{RESULTADOS E DISCUSSÃO}

Os filhotes machos apresentaram no mínimo 210 $\mathrm{mm}$ de CRC e no máximo $390 \mathrm{~mm}(\bar{x}=326,88$, ep $=$ $50,23, \mathrm{n}=17$ ), enquanto a menor fêmea mediu $215 \mathrm{~mm}$ e a maior $380 \mathrm{~mm}(\bar{x}=313,67$, ep $=49,04, \mathrm{n}=15)$. Os jovens machos apresentaram no mínimo $400 \mathrm{~mm}$ de CRC e no máximo $735 \mathrm{~mm}$, enquanto a menor fêmea $405 \mathrm{~mm}$ e a maior $690 \mathrm{~mm}$. Os adultos machos mediram entre 500 a $860 \mathrm{~mm}$ e as fêmeas entre 720 e $1040 \mathrm{~mm}$ de CRC (Tabs I, II). Sazima (1992) registrou fêmeas maduras com $750 \mathrm{~mm}$, similarmente a este estudo. JANEIRO-CINQUINI (2004) obteve uma fêmea madura com $620 \mathrm{~mm}$ de CRC.

Com o intuito de comparar as médias de tamanho das 14 variáveis morfométricas em função da classificação etária e sexual, utilizou-se análise de variância (ANOVA). Onze variáveis revelaram diferenças significativas nas médias entre machos e fêmeas na idade adulta, indicando dimorfismo sexual (CRC, CT, LCA, LO, LN, LL, DON, DLN, CA, DRL, DVS). Além de demonstrarem diferenças nas médias entre os sexos na idade adulta, a DLN e a DON apresentaram proximidade nas médias entre adultos machos e jovens fêmeas, da mesma forma a DLN possui proximidade entre filhotes fêmeas e jovens machos; para DOL, machos adultos e jovens não diferem, mostrando que esta medida se estabiliza antes do amadurecimento. As comparações entre machos e fêmeas quando filhotes apresentaram proximidade nas médias em todas variáveis, indicando que não há dimorfismo sexual (Fig. 6). Esse comportamento distinto entre variáveis e entre os sexos pode indicar (1) que fêmeas crescem mais rapidamente antes da maturidade para estas variáveis (DLN, DON, DOL), (2) que machos atingem a maturidade mais cedo, ou (3) ambas as coisas. 

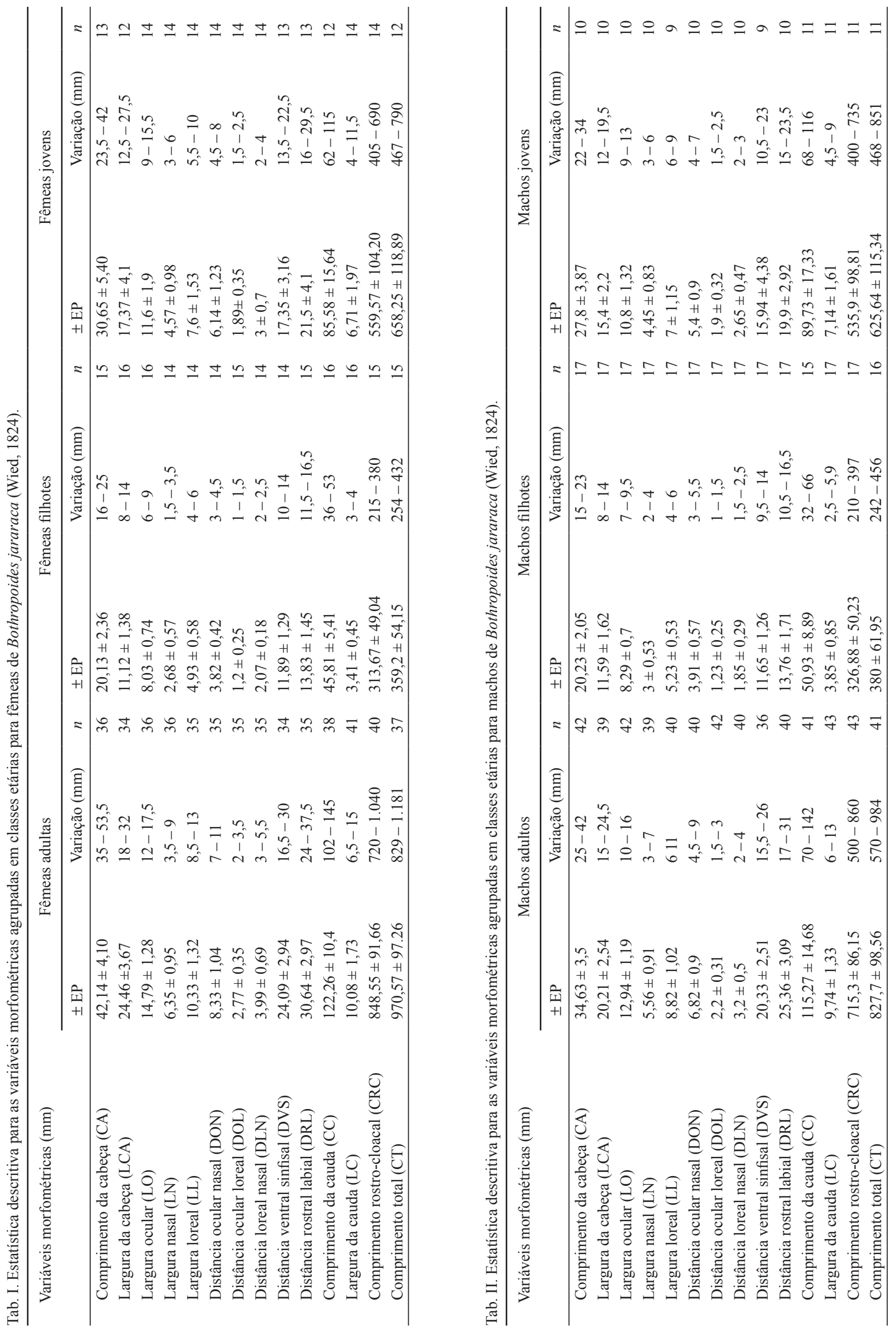

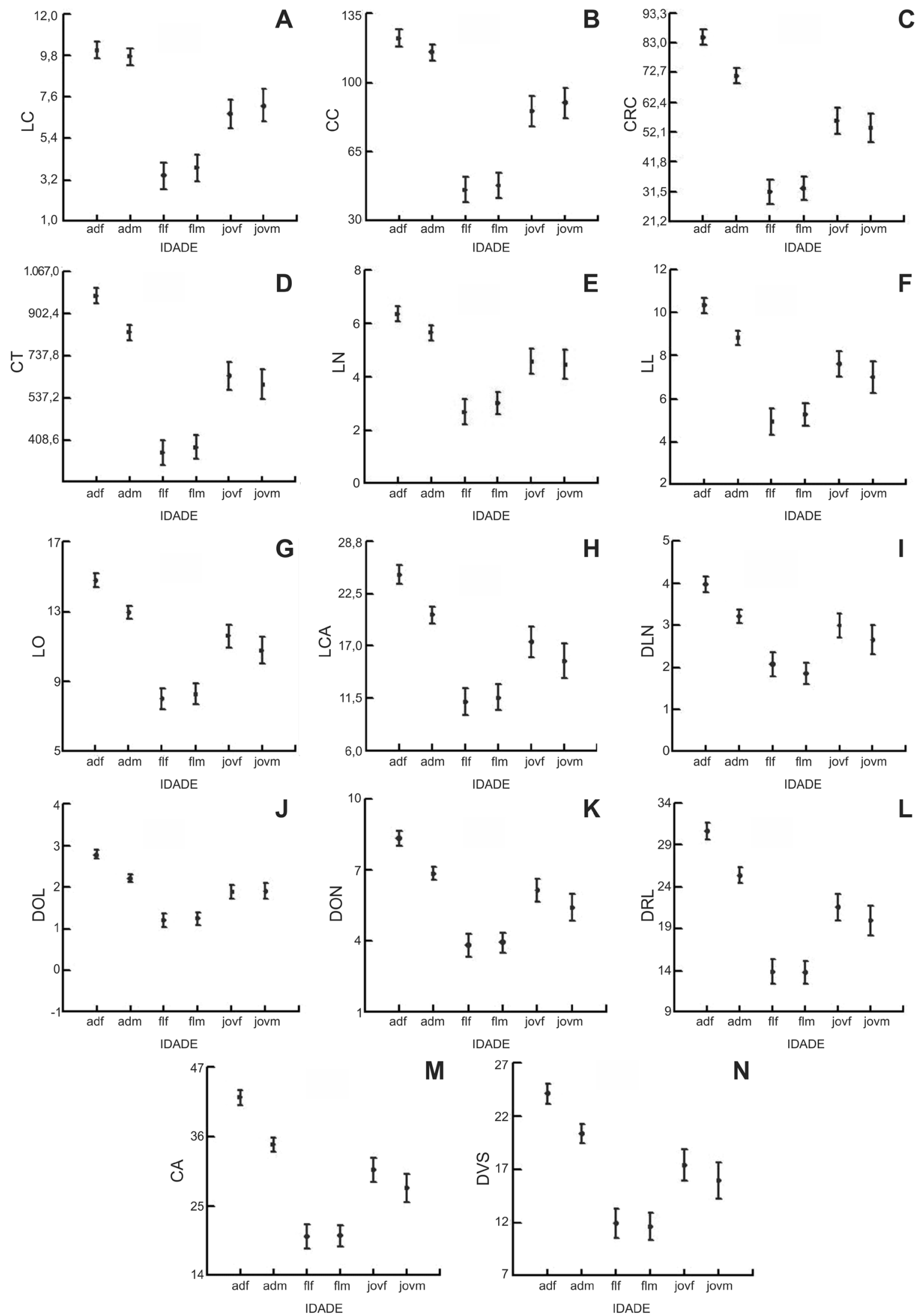

Fig. 6. ANOVA para as variáveis morfométricas de Bothropoides jararaca (Wied, 1824) agrupadas em classes etária e sexual: A, largura da cauda (LC); B, comprimento da cauda (CC); C, comprimento rostro-cloacal (CRC); E, comprimento total (CT); E, largura nasal (LN); F, largura loreal (LL); G, largura ocular (LO); H, largura da cabeça (LCA); I, distância loreal nasal (DLN); J, distância ocular loreal (DOL); K, distância ocular nasal (DON); L, distância rostral labial (DRL); M, comprimento da cabeça (CA); N, distância ventral sinfisial (DVS). 

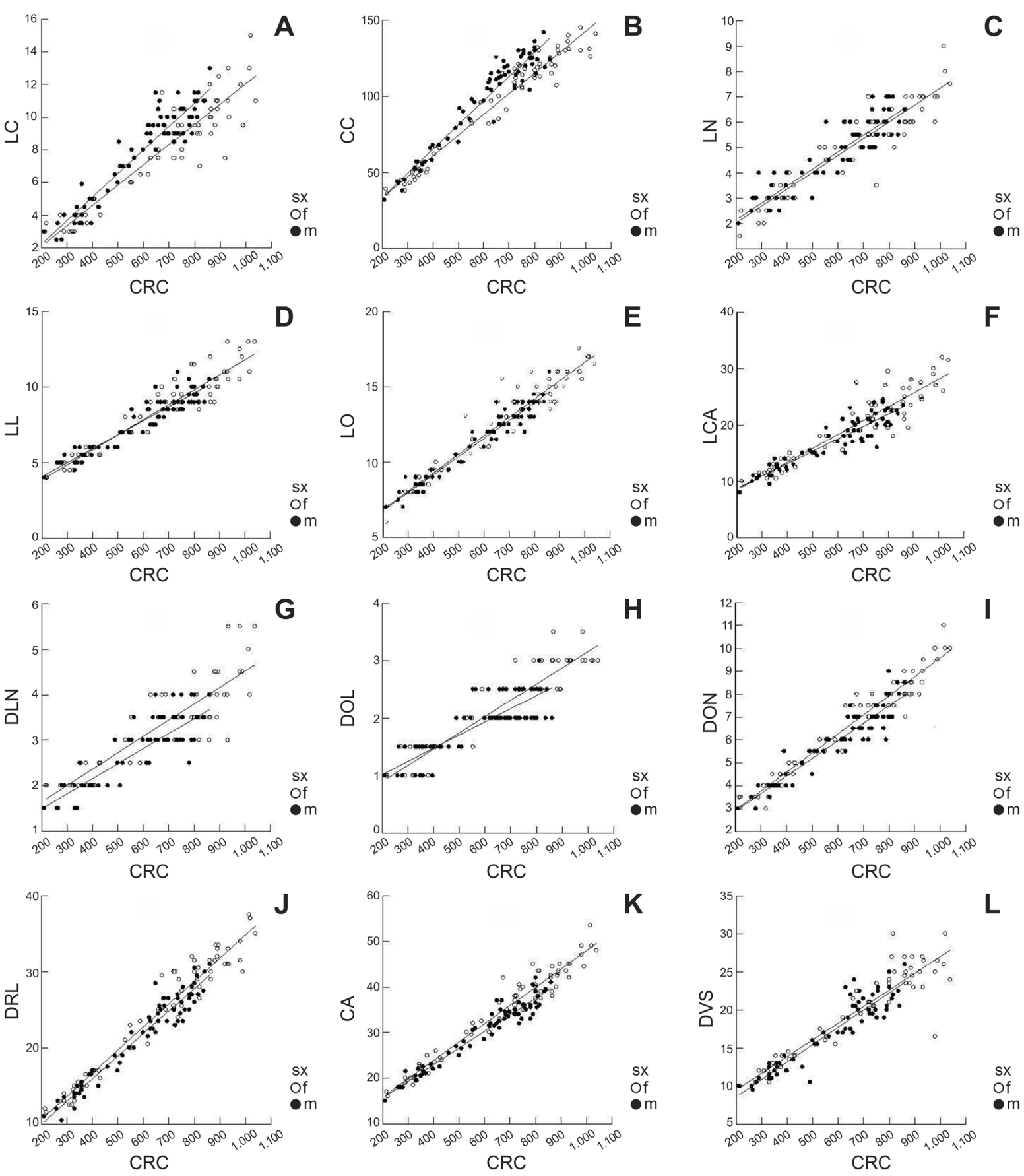

Fig. 7. Regressão linear para as variáveis morfométricas em função do comprimento rostro-cloacal (CRC) para fêmeas (f) e machos (m) de Bothropoides jararaca (Wied, 1824): A, largura da cauda (LC); B, comprimento da cauda (CC); C, largura nasal (LN); D, largura loreal (LL); E, largura ocular (LO); F, largura da cabeça (LCA); G, distância loreal nasal (DLN); H, distância ocular loreal (DOL); I, distância ocular nasal (DON); J, distância rostral labial (DRL); K, comprimento da cabeça (CA); L, distância ventral sinfisial (DVS).

Com o objetivo de compreender o dimorfismo morfométrico ontogenético de Botropoides jararaca utilizou-se regressão linear, testando todas as variáveis em função do CRC $(P<0,001)$. Em todas as medidas as fêmeas crescem mais que os machos. Porém, existem particularidades em algumas variáveis: CA, LCA, LO, LN, DON, DVS e DRL que apresentaram similaridade entre os sexos no início da vida e posteriormente machos parecem crescer mais lentamente que as fêmeas. Para CC e LC ocorre o inverso, machos crescem mais rapidamente que as fêmeas. No que se refere a DLN, os machos são menores que as fêmeas desde os estágios iniciais de vida e parecem apresentar uma taxa de crescimento mais lenta. Para as medidas das fossetas loreais (LL, DOL) observa-se que machos foram maiores quando filhotes e posteriormente fêmeas cresceram mais rapidamente (Fig. 7).

A variável ADC foi relativamente exitosa em recuperar os indivíduos pré-classificados em classes sexuais e etárias como grupos. Percebe-se que, no 
Tab. III. Coeficientes de regressão linear em função do comprimento rostro-cloacal (CRC) para machos e fêmeas de Bothropoides jararaca (Wied, 1824).

\begin{tabular}{lcc}
\hline Medidas & \multicolumn{1}{c}{ Machos } & Fêmeas \\
\hline Comprimento da cabeça (CA) & $\mathrm{p}<0,001 ; \mathrm{r}^{2}=0,941$ & $\mathrm{p}<0,001 ; \mathrm{r}^{2}=0,941$ \\
Largura da cabeça (LCA) & $\mathrm{p}<0,001 ; \mathrm{r}^{2}=0,856$ & $\mathrm{p}<0,001 ; \mathrm{r}^{2}=0,865$ \\
Largura ocular (LO) & $\mathrm{p}<0,001 ; \mathrm{r}^{2}=0,936$ & $\mathrm{p}<0,001 ; \mathrm{r}^{2}=0,932$ \\
Largura nasal (LN) & $\mathrm{p}<0,001 ; \mathrm{r}^{2}=0,821$ & $\mathrm{p}<0,001 ; \mathrm{r}^{2}=0,855$ \\
Largura loreal (LL) & $\mathrm{p}<0,001 ; \mathrm{r}^{2}=0,884$ & $\mathrm{p}<0,001 ; \mathrm{r}^{2}=0,899$ \\
Distância ocular-nasal (DON) & $\mathrm{p}<0,001 ; \mathrm{r}^{2}=0,886$ & $\mathrm{p}<0,001 ; \mathrm{r}^{2}=0,940$ \\
Distância ocular-loreal (DOL) & $\mathrm{p}<0,001 ; \mathrm{r}^{2}=0,692$ & $\mathrm{p}<0,001 ; \mathrm{r}^{2}=0,876$ \\
Distância loreal-nasal (DLN) & $\mathrm{p}<0,001 ; \mathrm{r}^{2}=0,696$ & $\mathrm{p}<0,001 ; \mathrm{r}^{2}=0,776$ \\
Distância ventral-sinfisial (DVS) & $\mathrm{p}<0,001 ; \mathrm{r}^{2}=0,866$ & $\mathrm{p}<0,001 ; \mathrm{r}^{2}=0,857$ \\
Distância rostral-labial (DRL) & $\mathrm{p}<0,001 ; \mathrm{r}^{2}=0,937$ & $\mathrm{p}<0,001 ; \mathrm{r}^{2}=0,949$ \\
Comprimento da cauda (CC) & $\mathrm{p}<0,001 ; \mathrm{r}^{2}=0,926$ & $\mathrm{p}<0,001 ; \mathrm{r}^{2}=0,947$ \\
Largura da cauda (LC) & $\mathrm{p}<0,001 ; \mathrm{r}^{2}=0,893$ & $\mathrm{p}<0,001 ; \mathrm{r}^{2}=0,867$ \\
Comprimento total (CT) & $\mathrm{p}<0,001 ; \mathrm{r}^{2}=0,998$ & $\mathrm{p}<0,001 ; \mathrm{r}^{2}=0,999$ \\
\hline
\end{tabular}

Tab. IV. Funções discriminantes canônicas, eigenvalues e porcentagem da variância explicada pelas funções, a partir da ADC de 14 variáveis morfométricas e 142 exemplares classificados em 6 grupos etários e sexuais.

\begin{tabular}{lcccc}
\hline \multirow{2}{*}{ Variáveis morfométricas } & \multicolumn{4}{c}{ Função Discriminante Canônica } \\
\cline { 2 - 5 } & 1 & 2 & 3 & 4 \\
\hline Comprimento da cabeça (CA) & $-0,002$ & 1,597 & 1,595 & 0,013 \\
Largura da cauda (LC) & $-0,059$ & $-0,895$ & 0,064 & 1,444 \\
Comprimento rostro cloacal (CRC) & $-1,460$ & 7,196 & $-6,627$ & 7,392 \\
Comprimento Total (CT) & 2,495 & $-7,829$ & 5,090 & $-8,406$ \\
\hline Eigenvalue & 6,314 & 1,269 & 0,042 & 0,008 \\
\% variância explicada & 82,7 & 16,6 & 0,6 & 0,1 \\
\hline
\end{tabular}

início do desenvolvimento, os grupos apresentam maior sobreposição, que tende a diminuir ao longo do desenvolvimento ontogenético dos indivíduos (Fig. 8). O significado biológico das classes torna-se evidente, já que $79,2 \%$ dos casos foram considerados corretamente classificados. A ADC gerou quatro funções discriminantes

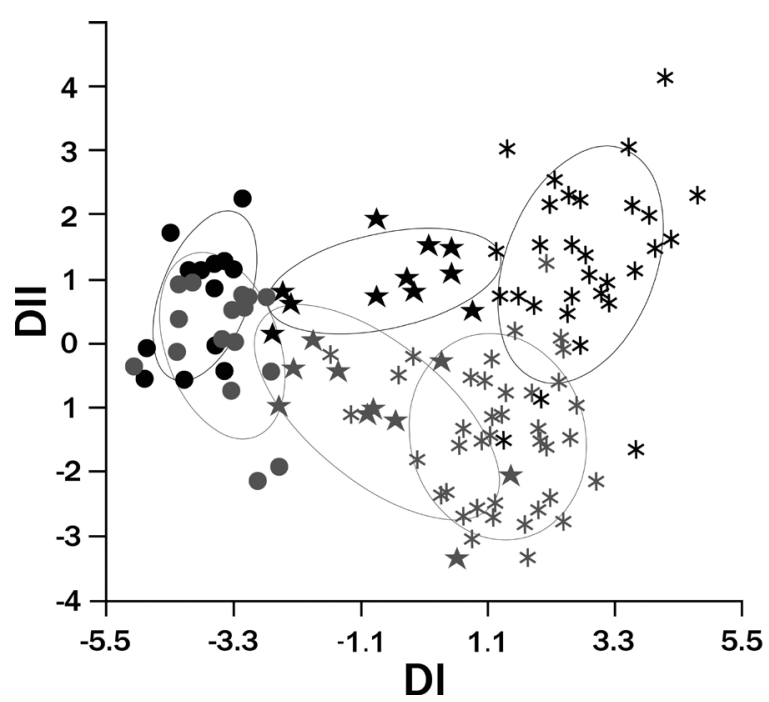

Fig. 8. Análise discriminante canônica para as classes etária e sexual de Bothropoides jararaca (Wied, 1824) (fêmeas em preto; machos em cinza; asterisco, adultos; estrela, jovens; círculo, filhotes). com $82,7 \%, 16,6 \%, 0,6 \%$ e $0,1 \%$ da variância grupada, respectivamente, a partir de quatro variáveis morfométricas (stepwise: CT, CRC, CA e LC) (Tab. III).

Conclui-se que o desenvolvimento ontogenético morfométrico de serpentes Botropoides jararaca no Rio Grande do Sul ocorre de formas diferentes para machos e fêmeas, com comportamentos distintos para as variáveis morfométricas utilizadas, principalmente DLN, CC e LC.

Agradecimentos. Agradecemos ao CNPq pela concessão da bolsa PIBIC; ao Márcio Borges Martins (Depto de Zoologia, UFRGS) pelas sugestões e ao Jean Pierre Corseuil pelas ilustrações.

\section{REFERÊNCIAS BIBLIOGRÁFICAS}

Alves, M. L. M.; Araujo, M. L. DE \& Witt, A. A. 2000. Aspectos da biologia reprodutiva de Bothrops jararaca em cativeiro (Serpentes: Viperidae). Iheringia, Série Zoologia (89):187-192.

Clark JR., D. R. 1966. Notes on sexual dimorphism in tail-length in American snakes. Transactions of Kansas Academy of Sciences 69:226-232.

Faria, R. G. \& Brites, V. L. C. 2003. Aspectos taxonômicos e ecológicos de Bothrops moojeni Hoge, 1966 (Serpentes: Crotalinae) do Triângulo e Alto Parnaíba, Minas Gerais, Brasil. Biologia Geral e Experimental 3(2):25-32.

Fenwick, A. M.; Gutberlet Jr., R. L.; Evans, J. A. \& Parkinson, C. L. 2009. Morphological and molecular evidence for phylogeny and classification of South American pitvipers, genera Bothrops, Bothriopsis, and Bothrocophias (Serpentes: Viperidae). Zoological Journal of the Linnean Society 156:617-640. 
Fiтch, H. S. 1981. Sexual size differences in reptiles. University of Kansas Museum of Natural History Miscellaneous Publications 70:1-72.

Gomes, N. \& Puorto, G. 1993. Atlas Anatômico de Bothrops jararaca Wied, 1824 (Serpentes: Viperidae). Memórias do Instituto Butantan 55(1):69-100.

Hartmann, P. A.; Hartmann, M. T. \& Giasson, L. O. M. 2003. Uso do hábitat e alimentação em juvenis de Bothrops jararaca (Serpentes: Viperidae) na Mata Atlântica do sudeste do Brasil. Phyllomedusa 2(1):35-41.

JANEIRO-CINQUINI, T. R. F. 2004. Capacidade reprodutiva de Bothrops jararaca (Serpentes, Viperidae). Iheringia, Série Zoologia 94(4):429-431.

Janeiro-Cinquini, T. R. F.; Leinz, F. F. \& Figueiredo, V. C. F. 1992. Sexual Dimorphism in Adult Bothrops jararaca. Bulletin of the Chicago Herpetological Society 27(4):94-95.

Janeiro-Cinquini, T. R. F.; Leinz, F. F. \& IshizuKA, M. M. 1990. Body sizelittler relationships and some characteristics of litters in Bothrops jararaca. Bulletin of the Chicago Herpetological Society 25(5):84-85.

Jordão, R. DOS S. \& BizerRA, A. F. 1996. Reprodução, dimorfismo sexual e atividade de Simophis rhinostoma (Serpentes: Colubridae). Revista Brasileira de Biologia 56(3):507-512.

KeOGH, J. S. \& Wallach, V. 1999. Allometry and sexual dimorphism in the lung morphology of prairie rattlesnakes Crotalus viridis viridis. Amphibia-Reptilia 20:377-389.

KING. R. B. 1989. Sexual dimorphism in snake tail: length: sexual selection, natural selection, or morphological constraint? Biological Journal of Linnean Society 38:133-154.

Kissner, K. J.; Secoy, D. M. \& Forbes, M. R. 1998. Sexual dimorphism in size of cloacal glands of garter snake, Thamnophis radix haydeni. Journal of Herpetology 32:268-270.

KLauber, L. M. 1943. Tail-length differences in snakes with notes on sexual dimorphism and the coefficient of divergence. Bulletin of Zoological Society of San Diego 18:1-60.

Lema, T. 1994. Lista comentada dos répteis do Rio Grande do Sul. Comunicações do Museu de Ciências e Tecnologia da PUCRS, Série Zoologia 7:41-150.

Martins, M.; Araujo, M. S.; Sawaya R. J. \& Nunes, R. 2001. Diversity and evolution of macrohabitat use, body size and morphology in a monophyletic group of Neotropical pitvipers (Bothrops). Journal of Zoology 254:529-538.

Mesquita, D. O. \& Brites, V. L. C. 2003. Aspectos taxonômicos e ecológicos de uma população de Bothrops alternatus Duméril, Bibron \& Duméril, 1854 (Serpentes: Viperidae) das regiões do Triângulo e Alto Parnaíba, Minas Gerais. Biologia Geral e Experimental 3(2):33-38.
SAntos JR., A. P. dos \& Ribeiro, F. R. V. 2005. Dimorfismo sexual em uma prole da cobra d'água Helicops polyleps Gunther, 1861 (Serpentes: Colubridae) do oeste do estado do Pará, Brasil, como comentários sobre o período reprodutivo. Comunicações do Museu de Ciências e Tecnologia da PUCRS, Série Zoologia 18(1):67-71.

SAzImA, I. 1992. Natural history of the jararaca pitviper, Bothrops jararaca, in Southeastern Brazil. In: CAmpBell, J. A. \& Brodies JR., E. D. eds. Biology of the Pitvipers. Selva Press, Tyler, Texas. p.199-216.

Semlitsch, R. D. \& Gibbons, J. W. 1982. Body size dimorphism and sexual selection in two species of water snakes. Copeia 1982:974976.

SHINE, R. 1977a. Reproduction in Australian elapid snakes I. Testicular cycles and mating seasons. Australian Journal of Zoology 25:647-653.

1977b. Reproduction in Australian elapid snakes II. Female reproductive cycles. Australian Journal of Zoology 25:655-666. 1990. Proximate det erminants of sexual differences in adult body size. The American Naturalist 135:278-283.

1993. Sexual dimorphism in snakes. In: Seigel, R. A.; Collins, J. T. \& NovaK, S. S. eds. Snakes ecology and behavior. New York, McGraw-Hill. p. 49-86. 346 .

. 1994. Sexual dimorphism in snakes revised. Copeia 1994:326-

SBH Sociedade Brasileira de Herpetologia. 2010. Brazilian reptiles - List of species. Disponível em: <http://www.sbherpetologia. org.br>. Acesso em 20.01.2010.

2007. Herpetologia no Brasil II. Belo Horizonte, Sociedade Brasileira de Herpetologia. 202p.

Solórzano, A. \& Cerdas, L. 1987. Biologia reproductiva de la cascabel centroamericana Crotalus durissus durissus (Serpentes: Viperidae) en Costa Rica. Revista de Biologia Tropical (36):221226.

1989. Reproductive biology and distribution of the terciopelo, Bothrops asper (Serpentes: Viperidae) in Costa Rica. Herpetologica 45(4):444-450.

Stamps, J. A. 1993. Sexual size dimorphism in species with asymptotic growth after maturity. Biological Journal of the Linnean Society 50:123-145.

Zug, G. R.; Vitt, L. J. \& Caldwell, J. P. 2001. Herpetology an introductory biology of amphibians and reptiles. 2ed. San Diego, Academic Press. 630p.

Recebido em 12 de julho de 2010. Aceito em 13 de outubro de 2011. ISSN 0073-4721

Artigo disponível em: www.scielo.br/isz

Impresso e distribuído em 2012. 